\title{
WASP-12b as a prolate, inflated and disrupting planet from tidal dissipation
}

\author{
Shu-lin $\mathrm{Li}^{1,2}$, N. Miller ${ }^{3}$, Douglas N. C. Lin ${ }^{1,3, *}$, Jonathan J. Fortney ${ }^{3}$ \\ ${ }^{1}$ Kavli Institute for Astronomy and Astrophysics, Peking University Beijing,100871, China \\ ${ }^{2}$ Department of Astronomy, Peking University, Beijing 100871, China \\ ${ }^{3}$ Department of Astronomy and Astrophysics, University of California, Santa Cruz 95064, USA
}

The class of exotic Jupiter-mass planets that orbit very close to their parent stars were not explicitly expected before their discovery ${ }^{1}$. The recently found ${ }^{2}$ transiting planet WASP-12b has a mass $M_{p}=\mathbf{1 . 4}( \pm 0.1)$ Jupiter masses $\left(M_{J}\right)$, a mean orbital distance of only 3.1 stellar radii (meaning it is subject to intense tidal forces), and a period of 1.1 days. Its radius $\mathbf{1 . 7 9}( \pm 0.09) R_{J}$ is unexpectedly large and its orbital eccentricity $\mathbf{0 . 0 4 9}( \pm 0.015)$ is even more surprising as such close orbits are in general quickly circularized. Here we report an analysis of its properties, which reveals that the planet is losing mass to its host star at a rate $\sim 10^{-7} M_{J} \mathbf{y r}^{-1}$. The planet's surface is distorted by the star's gravity and the light curve produced by its prolate shape will differ by about ten per cent from that of a spherical planet. We conclude that dissipation of the star's tidal perturbation in the planet's convective envelope provides the energy source for its large volume. We predict up to $10 \mathrm{mJy}$ CO band-head (2.292 $\mu \mathrm{m})$ emission from a tenuous disk around the host star, made up of tidally stripped planetary gas. It may also contain a detectable resonant super-Earth, as a hypothetical perturber that continually stirs up WASP-12b's eccentricity. 
Gas giant planets contract as they age and cool. Theoretical models ${ }^{3,4}$ predict an upper radius limit $\simeq 1.2 R_{J}$ for mature Jupiter-like gas giants. The radii $\left(R_{p}\right)$ of $\sim 60$ short-period (mostly $P<5$ days) gas giants have been measured from transit light curves. In the mass range $M_{p} \simeq$ $(1 \pm 0.5) M_{J}$, most planets' $R_{p} \simeq(1.2 \pm 0.2) R_{J}$. However, several planets have observed $R_{p} \sim$ $1.5-1.8 R_{J}$. The rate of heat loss from these planets increases with their radius. The over-sized WASP-12b has an intrinsic radiative luminosity of $L_{p} \sim 1-2 \times 10^{28} \mathrm{erg} \mathrm{s}^{-1}$. If gravity provides the only source with which to replenish this heat loss, WASP-12b would contract ${ }^{3,5}$ significantly over 100 million years. The small orbital separation from its host star means that WASP-12b is one of the most intensely heated planets known. Stellar photons deposit energy onto a planet's day side, and reduce the internal heat $\operatorname{loss}^{6}$, but the absorbed stellar irradiation is efficiently re-radiated along with the heat flux from a planet's interior on its night side ${ }^{7}$. Although surface evaporation enlarges a planets tenuous atmosphere ${ }^{8}$, it cannot alone significantly modify its internal structure and evolution ${ }^{9}$.

To account for the inflated sizes of gas giants that are gigayears old, we consider additional heating mechanisms. Given its measured eccentricity $e_{p} \simeq 0.05$ and its proximity to its host star, one potential suitable energy source for WASP-12b (and other close-in planets) is the dissipation of the stellar tidal disturbance ${ }^{10}$ well below its photosphere. A planet's tidal heating rate $\dot{E}_{t} \sim$ $e^{2} G M_{p} M_{*} / a \tau_{e}$ (where $G$ is the gravitational constant and $a$ is the semi-major axis.) is determined by its circularization time scale $\tau_{e}$ (where subscript $e$ indicates eccentricity damping), which is proportional to its tidal quality factor ${ }^{11}, Q_{p}^{\prime}$. (Here $\mathbf{M}^{*}$ is the stellar mass.) We note that many long-period planets have elongated orbits $\left(0<e_{p}<1\right)$ whereas those of short-period gas giants 
are mostly circular (within the detection limit, $e_{p}<0.02$ ). If this transition is due to their own life-long tidal dissipation, their inferred $Q_{p}^{\prime} \sim 10^{6} t o 10^{7}$.

For its orbit, WASP-12b's $\tau_{e} \sim 0.033 Q_{p}^{\prime}\left(X_{R} / R_{p}\right)^{5}$ years, where $X_{R} \equiv\left(M_{p} / 3 M_{*}\right)^{1 / 3} a$ is the distance from the planet's core to its inner Lagrangian $\left(L_{1}\right)$ point (that is, the Roche radius). If WASP-12b's $Q_{p}^{\prime} \sim 10^{7}$, then its eccentricity would be damped within ten million years and the associated tidal heating rate would balance its current intrinsic radiative luminosity (that is, $\dot{E}_{t} \sim L_{p}$ ). Depending on their internal structure and orbital periods, the magnitude of $Q_{p}^{\prime}$ of gas giants and their host stars are thought to vary over a large magnitude range ${ }^{12,13}$ (see below). If WASP-12b's $Q_{p}^{\prime}$ is comparable to that inferred for Jupiter ${ }^{14}\left(\sim 10^{6}\right)$, its tidal heating would overcompensate beyond its intrinsic luminosity and would drive ongoing envelope expansion. Once the planet's atmosphere filled its Roche lobe, mass would be lost at a rate of $\dot{M}_{p} \sim \dot{E}_{t} R_{p} / 2 G M_{p}$.

To show that WASP-12b is currently losing mass, we analyse the amount of stellar light blocked during its primary transit by its cross-section in the (Y-Z) direction normal to the line (X) joining it and its host star (see Fig. 1). At the planet's occultation radius $R_{p}$ and maximum Y distance $\left(Y_{R}=2 X_{R} / 3\right)$ on the planet's Roche lobe, we estimate the atmosphere density to be $\rho\left(R_{p}\right) \sim 5 \times 10^{-8} \mathrm{~g} \mathrm{~cm}^{-3}$ and $\rho\left(Y_{R}\right) \sim 3 \times 10^{-12} \mathrm{~g} \mathrm{~cm}^{-3}$ respectively (see Supplementary Information). An interesting consequence of the planet's prolate shape is that, as a function of orbital phase, it will scatter incident stellar flux, and emit its own thermal radiation, differently than a spherical planet would. We estimate that this effect leads to 10Optical and mid-infrared orbitmodulated flux has been detected from several planets ${ }^{15}$ and WASP-12b is a promising candidate 
for detection as well.

Near $\mathrm{L}_{1}$, gas falls towards the host star through a nozzle with a radius $\Delta Y \sim 0.22 X_{R}$ (see Supplementary Information). The resultant pressure gradient on the Roche lobe drives gas from other regions to flow towards $L_{1}$. This advection along equipotential surfaces offsets the hydrostatic equilibrium normal to these surfaces. Consequently, the planet's lower atmosphere expands (see Fig. 1) at the sound speed $\left(c_{\text {sound }}\right)$ across the Roche lobe (which has a total surface area $\left.A_{R} \sim 4 \pi X_{R} Y_{R}\right)$ with a mass flux $\dot{M}_{\text {observed }}=\rho\left(Y_{R}\right) c_{\text {sound }} A_{R} \sim 10^{-7} M_{J} \mathrm{yr}^{-1}$. If WASP12b's $Q_{p}^{\prime} \sim 10^{6}$, its $\dot{M}_{p} \sim \dot{M}_{\text {observed }}$ and its atmosphere loss would be continually replenished by the tidal inflation of its envelope.

Gas slightly beyond the nozzle accelerates towards the star and attains a free fall speed $\left(v_{f}\right)$ and a local density $\rho\left(L_{1}\right) \simeq \rho\left(Y_{R}\right)\left(c_{\text {sound }} A_{R} / v_{f} \pi \Delta Y^{2}\right) \sim \rho\left(Y_{R}\right)$. Since $\rho\left(L_{1}\right)<<\rho\left(R_{p}\right)$, this stream remains optically thin. It does not directly strike WASP-12 but forms a disk which is tidally truncated $^{16}$ by WASP-12b at $\sim 0.7 a=3 R_{\odot}$. Provided WASP-12's magnetic field has a subsolar strength, the disk extends to the stellar surface and intercepts a significant fraction $(\sim 0.1)$ of the stellar visual luminosity ${ }^{17}$. Viscous stress also generates heat at a rate $\sim 10^{-2} L_{\odot}$ while it transfers mass and angular momentum ${ }^{18}$ (with an efficiency factor $\alpha \sim 10^{-2}-10^{-3}$ ) to produce a disk surface density $\Sigma \sim \alpha^{-1} \mathrm{~g} \mathrm{~cm}^{-2}$.

The disk's warm $(\sim 3000-4000 \mathrm{~K})$ midplane is sandwiched by heated surface layers which emit continuum and line radiation, respectively. At these temperatures, $\mathrm{CO}$ molecules are preserved and their 2-0 band-head emission at $\sim 2.292 \mu \mathrm{m}(1-10 \mathrm{mJy}$ at WASP-12's 267 pc distance 
from the Sun) is comparable to that of their continuum ${ }^{19}$. Although the expected flux is 1-2 orders of magnitude below that found around some known young stellar objects, this disk signature may be marginally observable with existing near infrared spectrometers. Pollution of a few Earth masses of metals may also enhance the $[\mathrm{Fe} / \mathrm{H}]$ of star's low-mass $\left(<10^{-3} M_{\odot}\right)$ convection zone depending on the efficiency of a double diffusive instability ${ }^{20}$.

As its tidal debris flows through the disk onto the surface of its host star, WASP-12b gains orbital angular momentum. Its orbit also exchanges angular momentum with its host star at a rate which is regulated by the dissipative efficiency of its tidal perturbation within the stellar envelope $^{21,22}$. WASP-12b would spiral toward its host star on a time scale $\tau_{a} \sim 10 \mathrm{Myr}$ if the quality factor $\left(Q_{*}^{\prime}\right)$ of its slowly spinning host star is comparable to that $\left(\sim 10^{6}\right)$ estimated from the circularization periods of binary stars in open clusters ${ }^{23}$.

The low probability of catching a brief glimpse of WASP-12b's rapid orbital evolution and mass loss can be circumvented with variable $Q_{*}^{\prime}$-values ${ }^{13}$. We suggest that during WASP-12's main sequence evolution, its $Q_{*}^{\prime}$ may have been larger than $10^{8}$ such that its planet's orbit did not evolve significantly. But, as it evolves onto its subgiant track, WASP-12's intrinsic oscillation frequencies are modified with the expansion of its radius, deepening of its convective zone, and slowdown of its spin. When these frequencies are tuned to match with those of the planet's tidal perturbation, the star's dynamical response is greatly amplified. In these resonant episodes, $Q_{*}^{\prime}$ may decrease ${ }^{13}$ below $\sim 10^{7}$, leading to the present epoch of intense star-planet tidal interaction and dissipation.

Our result for the mass loss from WASP-12b is robust, whereas the tidal heating scenario 
depends on the assumed $Q_{p}^{\prime}<10^{7}$ today. But, the preservation of WASP-12b's finite eccentricity implies that its $Q_{p}^{\prime}>10^{9}$ for most of its life. It is possible that during its recent accelerated orbital decay (due to a decline in $Q_{*}^{\prime}$ ), the evolving stellar perturbation frequency and strength may have intensified the planet's tidal response and dissipation over some ranges of its orbital period. The subsequent expansion of the heated envelope leads to further changes in the planet's oscillation frequencies and enlarges the range of efficient tidal dissipation. We estimate an effective $Q_{p}^{\prime}\left(\sim 10^{6-7}\right)$ for the present-day WASP-12b which is comparable to that needed to maintain its inflated size.

It is also possible that WASP-12b's eccentricity is continually excited by one or more superEarths with periods $P_{2}<P$ and masses $M_{2}<M_{p}\left(P_{2} / P\right)^{13 / 3}$. Their convergent paths would lead to resonant capture ${ }^{24}$ followed by lock-step orbital decay and $e$ growth to an equilibrium ${ }^{25}$. Episodic declines of $Q_{*}^{\prime}$ and $Q_{p}^{\prime}$ (over a few Myr) would excite WASP-12b's equilibrium eccentricity and inflate its radius to their observed values. Such a hypothetical planet would be embedded ${ }^{16}$ in the circumstellar disk and the orbital migration induced by its tidal interaction with the disk ${ }^{26}$ (on time scales $>10^{8} \mathrm{yr}$ ) would not affect its resonant interaction with WASP-12b. The sensitivity of its radial velocity detection would need to be $<1 \mathrm{~ms}^{-1}$ and its transit observation would need to be $10^{-1}$ magnitude.

Our analysis can be used to constrain the efficiency of tidal dissipation of other close-in planets such as the recently discovered ${ }^{22}$ massive $\left(10 M_{J}\right)$, short-period $(0.94 \mathrm{~d})$ WASP- $18 \mathrm{~b}$. If it and its host star have similar $Q^{\prime}$ values $\left(\sim 10^{6}\right)$, WASP-18b's tidal dissipation (at a rate $\sim 10^{30} \mathrm{erg}$ 
$\mathrm{s}^{-1}$ ) would maintain the synchronization of its $\operatorname{spin}^{10}$ and induce mass loss through Roche-lobe overflow during the decay of its orbit (induced by the star's tidal dissipation) on a time scale of $\sim 1$ Myr. However, WASP-18b is observed to have a normal radius $\left(1.1 R_{J}\right)$ for massive gas giants which places an upper limit $\left(<10^{28} \mathrm{erg} \mathrm{s}^{-1}\right)$ on the planetary dissipation rate ${ }^{5}$ which implies either $Q_{*}^{\prime}>10^{7}$ or $Q_{p}^{\prime}>10^{10}$ (or both). This constraint include the possibility for WASP-18b to be preserved (with $Q_{*}^{\prime}>10^{9}$ ) until the end of its host star's main sequence evolution.

\section{Supplementary Information}

Outside $R_{p}$, an optically thin atmosphere establishes a quasi hydrostatic equilibrium which extends to the planet's Roche-lobe surface. Neglecting small temperature, sound speed $\left(c_{s}\right)$, and opacity $\left(\kappa_{0}\right)$ variations, the gas density at a radial location $R^{\prime}\left(>R_{p}\right)$ is

$$
\rho\left(R^{\prime}\right)=\rho\left(R_{p}\right) \exp \left[-\lambda\left(1-R_{p} / R^{\prime}\right)\right]
$$

where $\lambda=G M_{p} / c_{s}^{2} R_{p}$. Assuming spherical symmetry, we find $\rho\left(R_{p}\right)=\left[\kappa_{0} f\left(\theta_{1}\right) R_{p}\right]^{-1}$ where

$$
f\left(\theta_{1}\right) \equiv \int_{-\theta_{1}}^{\theta_{1}}\left(d \theta / \cos ^{2} \theta\right) \exp [-\lambda(1-\cos \theta)]
$$

is a weak function of $\theta_{1} \equiv \cos ^{-1}\left(Y_{R} / R_{p}\right)$. WASP-12b's atmospheric temperature at $X_{R}$ is $\sim$ $2,500 \mathrm{~K}$ for effective planet-wide energy redistribution of absorbed stellar flux and $\sim 3000 \mathrm{~K}$ on the day-side for inefficient thermal circulation. A self-consistent analysis of transit data ${ }^{27}$ yields $\kappa \sim 10^{-2} \mathrm{~cm}^{2} \mathrm{~g}^{-1}$ at $R_{p}$. With corresponding values of $c_{s} \sim 5 \mathrm{~km} \mathrm{~s}^{-1}, \lambda=59$, and $f=0.165$ at pericenter (where $X_{R}=3.2 R_{J}$ and $Y_{R}=2.1 R_{J}$ ), we find $\rho\left(R_{p}\right) \sim 5 \times 10^{-8} \mathrm{~g} \mathrm{~cm}^{-3}$ and $\rho\left(Y_{R}\right) \sim 3 \times 10^{-12} \mathrm{~g} \mathrm{~cm}^{-3}$ respectively. 
On the Roche lobe, gas flows toward $\mathrm{L}_{1}$ and is channeled into a stream which falls toward the host star with a flux $\dot{M}_{o b s}$. Gas in the stream is confined by the Y opening of the equipotential surface which contains atmosphere at one unperturbed scale height $Y_{R} / \lambda=Y_{T} c_{s}^{2} R_{p} / G M_{p}$ outside $Y_{R}$. The radius of the nozzle is $\Delta Y=(2 / 3)^{1 / 2} c_{s} P / 2 \pi \sim 0.4 R_{p} \sim 0.22 X_{R}$. Despite the convergent flow, gas density along in the stream is $\sim \rho\left(Y_{R}\right)$ as its main flow velocity is accelerated to the supersonic free fall speed. Since $\rho\left(Y_{R}\right)<<\rho\left(R_{p}\right)$, this stream is optically thin.

We thank an anonymous referee for pointing out that the mass loss rate due to the X-ray and EUV driven thermal evaporation of the upper atmosphere is negligible compared with $\dot{M}_{\text {obs }}$. WASP-12 may also have a wind with a flux $\dot{M}_{w}$ and speed $v_{w}$ comparable to those of the Sun $\left(\sim 10^{-13} M_{\odot} \mathrm{yr}^{-1}\right.$ and $\left.<10^{3} \mathrm{~km} \mathrm{~s}^{-1}\right)$. The wind's ram pressure $\dot{M}_{w} v_{m} / 4 \pi a^{2}$ on WASP-12b's day side could potentially exceed the planet's atmospheric thermal pressure and perturb the flow toward the night $\operatorname{side}^{28}$ but it is inadequate to push the gas to overcome the gravitational potential difference $^{29} 2 G M_{*} / 3 a$ between $L_{1}$ and the outer Lagrangian point at $L_{2}$. The ongoing expansion of WASP-12b's envelope would continually increase its atmospheric density until a mass-flow equilibrium is established with an outflow through the $L_{1}$ nozzle. Finally, if WASP-12b has a magnetic field larger than a few Gauss, outflow of a tenuous, highly ionized atmosphere could be suppressed. However, the accumulation of the expanding envelope would also quench the magnetic influences in WASP-12b's atmosphere and lead to an outflow equilibrium.

1. Mayor, M. \& Queloz, D. A Jupiter-Mass Companion to a Solar-Type Star. Astrophys. J. 378, 355-359 (1995). 
2. Hebb, L. et al. WASP-12 b: the hottest transiting extra solar-planet yet discovered. Astrophys. J. 693, 1920-1928 (2009).

3. Bodenheimer, P., Laughlin, G. \& Lin, D. N. C. On the Radii of Extrasolar Giant Planets. Astrophys. J. 592, 555-563 (2003).

4. Fortney, J. J., Marley, M. S. \& Barnes, J. W. Planetary Radii Across Five Orders of Magnitude in Mass and Stellar Insolation: Application to Transits. Astrophys. J. 659, 1661-1672 (2007).

5. Bodenheimer, P., Lin, D. N. C. \& Mardling, R. A. On the Tidal Inflation of Short-Period Extrasolar Planets. Astrophys. J. 548, 466-472 (2001).

6. Guillot, T., Burrow, A., Hubbard, W. B., Lunine, J. I. \& Saumon, D. Giant Planets at Small Orbital Distances. Astrophys. J., 459, L35-L38 (1996).

7. Dobbs-Dixon, I. \& Lin, D. N. C. Atmospheric Dynamics of Short-Period Extrasolar Gas Giant Planets. I. Dependence of Nightside Temperature on Opacity. Astrophys. J., 673, 513-525 (2008).

8. Garcia Munoz, A., Physical and chemical aeronomy of HD 209458b. P\&SS 55, 1426-1455 (2007).

9. Hubbard, W. B., Hattori, M. F., Burrows, A., Hubeny, I. \& Sudarksy, D. Effects of mass loss for highly-irradiated giant planets. Icarus 187, 358-364 (2007).

10. Dobbs-Dixon, I., Lin, D. N. C. \& Mardling, R. A. Spin-Orbit Evolution of Short-period Planets. Astrophys. J. 610, 464-476 (2004). 
11. Goldreich, P. \& Soter, S. Q in the Solar System. Icarus, 5, 375-389 (1966).

12. Ogilvie, G. I. \& Lin, D. N. C. Tidal Dissipation in Rotating Giant Planets. ApJ 610, 477-509 (2004).

13. Ogilvie, G. I. \& Lin, D. N. C. Tidal Dissipation in Rotating Solar-Type Stars. Astrophys. J. 661, 1180-1191 (2007).

14. Yoder, C. F. \& Peale, S. J. The Tide of Io. Icarus 47, 1-35 (1981).

15. Knutson, H. et al. A map of the day-night contrast of the extrasolar planet HD 189733b. Nature 447, 183-186 (2007).

16. Lin, D. N. C. \& Papaloizou, J. C. B. On the tidal interaction between protostellar disks and companions. in Protostars and Planets III (eds: D. Black and M. Mathews, University of Arizona Press). 749-835 (1993).

17. Adams, F. C., Shu, F. H. \& Lada, C. J. The disks of T Tauri stars with flat infrared spectra. Astrophys. J. 326, 865-883 (1988).

18. Hartmann, L., Calvet, N., Gullbring, E. \& D'Alessio, P. Accretion and the Evolution of T Tauri Disks. Astrophys. J. 495, 385-400 (1998).

19. Najita, J., Carr, J. S., Glassgold, A. E., Shu, F. H. \& Tokunaga, A. T. Kinematic Diagnostics of Disks around Young Stars: CO Overtone Emission from WL 16 and 1548C27. Astrophys. J. 462, 919-936 (1996). 
20. Vauclair, S. Metallic Fingers and Metallicity Excess in Exoplanets' Host Stars: The Accretion Hypothesis Revisited. Astrophys. J. 605, 874-879 (2004).

21. Sasselov, D. D. The New Transiting Planet OGLE-TR-56b: Orbit and Atmosphere. Astrophys. J. 596, 1327-13312003.

22. Hellier, C., et al. An orbital period of 0.94days for the hot-Jupiter planet WASP-18b. Nature 460, 1098-1100 (2009).

23. Meibom, S., Mathieu, R. D. \& Stassun, K. G. An Observational Study of Tidal Synchronization in Solar-Type Binary Stars in the Open Clusters M35 and M34. Astrophys. J. 653, 621-635 (2006).

24. Peale, S., Cassen, P. \& Reynolds, R. T. Melting Io by Tidal Dissipation. Science 203, 892-894 (1979).

25. Lin, D. N. C. \& Papaloizou, J. On the structure of circumbinary accretion disks and the tidal evolution of commensurable satellites. Mon. Not. R. Astron. Soc. 188, 191-201 (1979).

26. Tanaka, H., Takeuchi, T. \& Ward, W. R. Three-Dimensional Interaction between a Planet and an Isothermal Gaseous Disk. I. Corotation and Lindblad Torques and Planet Migration. Astrophys. J. 565, 1257-1274 (2002).

Supplementary Information is linked to the online version of the paper at www.nature.com/nature.

Acknowledgements This work is supported by the Kavli Foundation which enabled the principle initiation and development of this work at KIAA-PKU. It is also supported by NASA, JPL, and NSF. 
Author contributions Shulin Li and D.N.C. Lin constructed arguments for mass loss and tidal heating of WASP-12b. They also composed the draft of the paper. N. Miller brought to the attention of the team on WASP-12b's large radius and designed the illustration. J. Fortney contributed information on the planet's opacity and improved the presentation of the manuscript.

Competing Interests The authors declare that they have no competing financial interests.

Author Information Correspondence and requests for materials should be addressed to Douglas N. C. Lin (email: lin@ucolick.org), Department of Astronomy and Astrophysics, University of California, 1156 High Street, Santa Cruz, CA 95064, Fax: 8314595265. 
Figure 1 WASP-12b's surfaces. The inner opaque purple surface (We note its prolate shape) contains the planet's envelope, which contributes to the eclipse of the stellar light, as inferred from transit observations. The stellar photons are represented by the orange arrows. The planet's outer transparent surface is the $L_{1}$ equal potential surface and its projection onto the orbital planet is contained in the solid purple line. The equipotential surfaces are computed assuming that the planet is a point mass on a nearly circular orbit. WASP-12b has a thin atmosphere that extends between these two surfaces; some of this atmosphere flows through the inner Lagrange point and eventually onto WASP-12. The purple dotted lines indicate the projection of the equipotential surface which channels the flow (purple arrows) from the planet's expanding envelope. The presence of a tenuous (light purple) disk with a hypothetical embedded planet (black dot) around WASP-12 (light yellow sphere) is illustrated at the top. The planet's orbit is traced by the purple dashed line. All variables are defined in the text. 


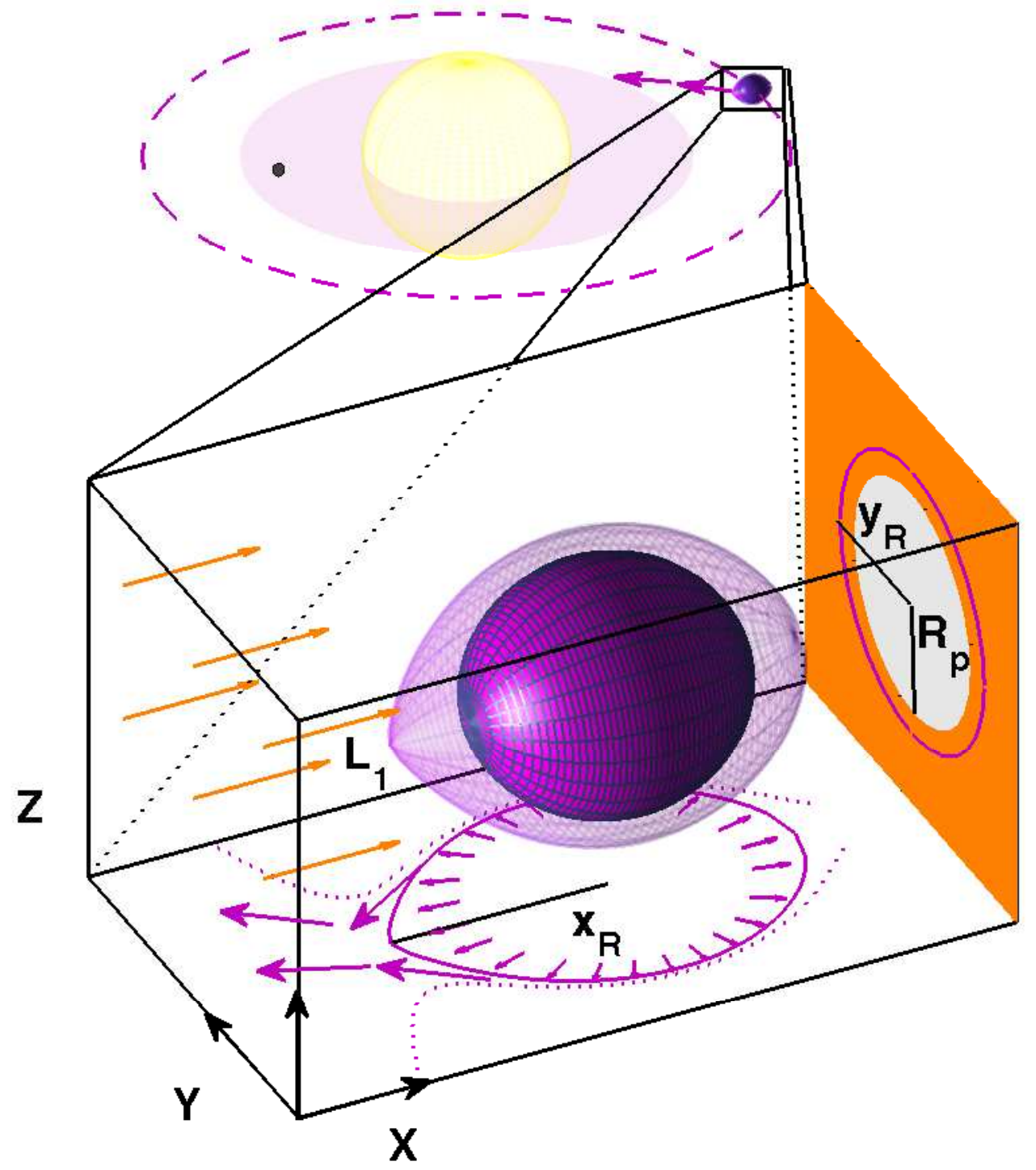

